\title{
Gold Nanorod Array-Bridged Internal-Standard SERS Tags: From Ultrasensitivity to Multifunctionality
}

\author{
Rongchao Mei, ${ }^{\dagger}$ Yunqing Wang, ${ }^{*}{ }^{\dagger}$ Qian Yu, ${ }^{\dagger}$ Yingchao Yin, ${ }^{\ddagger}$ Rongfang Zhao, ${ }^{\dagger}$ and Lingxin Chen ${ }^{*}, \dagger, \S^{\prime} \|_{\odot}$ \\ ${ }^{\dagger}$ CAS Key Laboratory of Coastal Environmental Processes and Ecological Remediation, Yantai Institute of Coastal Zone Research, \\ Chinese Academy of Sciences, Yantai 264003, China \\ ${ }^{\ddagger}$ School of Pharmacy, Collaborative Innovation Center of Advanced Drug Delivery System and Biotech Drugs in Universities of \\ Shandong, Key Laboratory of Molecular Pharmacology and Drug Evaluation (Yantai University), Ministry of Education, Yantai \\ University, Yantai 264005, China \\ ${ }^{\S}$ College of Chemistry and Chemical Engineering, Qufu Normal University, Qufu 273165, China \\ "Center for Ocean Mega-Science, Chinese Academy of Sciences, Qingdao 266071, China
}

\section{Supporting Information}

ABSTRACT: Bimetallic gold core-silver shell (Au@Ag) surfaceenhanced Raman scattering (SERS) tags draw broad interest in the fields of biological and environmental analysis. In reported tags, silver coating tended to smooth the surface and merge the original hotspot of $\mathrm{Au}$ cores, which was disadvantageous to signal enhancement from the aspect of surface topography. Herein, we developed gold nanorod (AuNR)-bridged Au@Ag SERS tags with uniform three-dimensional (3D) topography for the first time. This unique structure was achieved by selecting waxberry-like Au nanoparticles (NPs) as cores, which were capped by vertically oriented AuNR arrays. Upon selective surface blocking with thiolligands, Ag NPs were controlled to anisotropically grow on the tips of the AuNRs, producing high-density homo- $(\mathrm{Ag}-\mathrm{Ag})$ and hetero- $(\mathrm{Au}-\mathrm{Ag})$ hotspots in a single NP. The 3D hotspots rendered this NP extraordinary SERS enhancement ability (an analytical enhancement factor of $3.4 \times 10^{6}$ ) 30 times higher than the counterpart with a smooth surface, realizing signal detection from a single NP. More importantly, multiplexing signals ("blank" or multiplex "internal standard") can be achieved by simply changing thiol-ligands, as exemplified in the synthesis of NPs with 8 signatures. Furthermore, the multifunctionality has been demonstrated in living cell/in vivo imaging, photothermal therapy, and SERS substrates for ratiometric quantitative analysis, relying on the inherent internal standard signal. The prepared $\mathrm{Au} @ \mathrm{Ag}$ NPs have great potential as standard tools in many SERS-related fields.

KEYWORDS: SERS, gold nanorod, silver nanoparticle, bimetallic, internal standard

\section{INTRODUCTION}

As we all know, the sensitivity of surface-enhanced Raman scattering (SERS) detection is determined by noble metal nanoparticles (NPs). ${ }^{1-3}$ Recently, bimetallic silver shell-coated gold core $(\mathrm{Au} @ \mathrm{Ag})$ NPs have drawn remarkable interests as SERS nanosubstrates. ${ }^{4-6}$ They perfectly combine the superior properties of classic gold and silver NPs. On one hand, they have uniform morphology and tunable shapes (sphere, ${ }^{7}$ rod, $^{8,9}$ star, ${ }^{10}$ etc.) taking advantage of the Au NP cores with wellcontrolled morphologies. On the other hand, they perfectly inherit ideal signal enhancement ability of silver, illustrating much higher SERS detection sensitivity than Au NPs with similar size. ${ }^{3-6}$ More importantly, novel Raman reporterembedded Au@Ag NPs have been developed. ${ }^{11}$ This bimetallic structure gained strong and specific "internal standard (IS)" SERS signal by intercalating the Raman reporter between the $\mathrm{Au}$ core and the $\mathrm{Ag}$ layer. ${ }^{8}$ This IS signal can calibrate the signal of analytes adsorbed on the outer Ag layer surface, making the NPs powerful candidates for reliable quantitative analysis. ${ }^{12,13}$ This is an important advancement for SERS applications in real samples, which promotes the value of Au@Ag NPs.

To date, Au@Ag bimetallic NPs for SERS detection can be classified into two structures, that is, $\mathrm{Au} / \mathrm{Ag}$-adhesive type and $\mathrm{Au} / \mathrm{Ag}$-gapped type. The silver shell of the former was directly deposited on a bare gold core ${ }^{14}$ or a Raman reporter (such as 4-mercaptobenzoic acid, ${ }^{8}$ 4-aminothiophenol ${ }^{12}$ )-attached gold core. There were no apparent gaps between the two metals. To obtain the latter NPs, gold cores were premodified with singlestrand DNA, ${ }^{15}$ polyelectrolytes, ${ }^{7}$ and copolymers. ${ }^{16}$ Silver ions

Received: October 9, 2019

Accepted: December 23, 2019

Published: December 23, 2019 


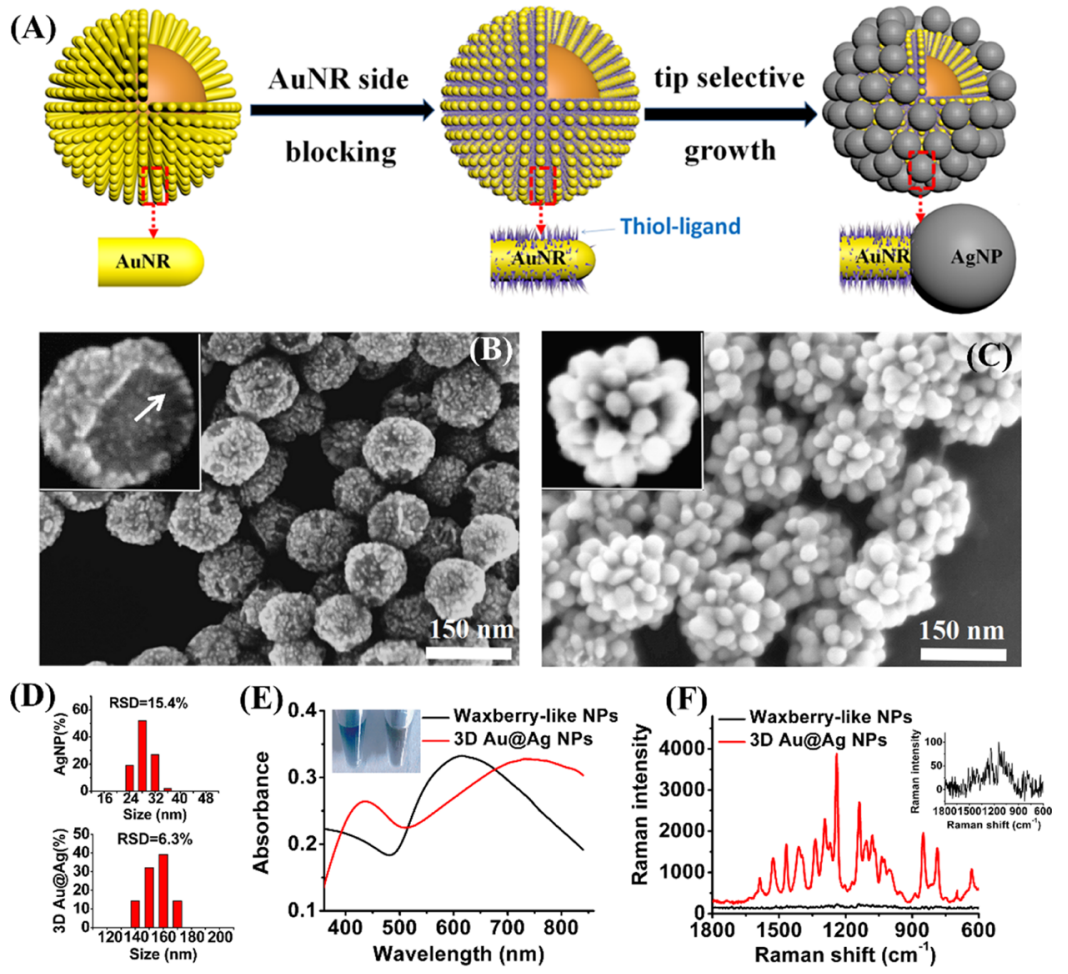

Figure 1. (A) Schematic fabrication of Au@Ag NPs with 3D topography. (B) SEM image of waxberry-like core NPs. The inset illustrates an elaborately selected core NP with an open pore on the core surface, in which vertically oriented AuNR arrays can be observed. (C) SEM image of 3D Au@Ag NPs prepared by using MEA as the blocking ligand. The inset shows a single NP covered by match-like NP arrays. (D) Size distribution of AgNPs and 3D Au@Ag NPs obtained by counting 100 of NPs. (E) UV/vis spectra and solution color of the waxberry-like core NPs (left) and 3D Au@Ag NPs (right). (F) SERS spectra of DTTC $\left(10^{-6} \mathrm{M}\right)$ as a reporter obtained from the waxberry-like core NPs and 3D Au@Ag NPs. The inset shows the spectrum from waxberry-like core on a small scale.

can be enriched and reduced over these molecules, leading to a middle gap within the resultant NPs. For both types of Au@Ag NPs, silver grew on the cores with high-density sites, thus easily fused, and smoothly covered the whole core surface.

It is well known that the surface topography of NPs plays an important role in the Raman enhancement ability. Rough NPs have much greater enhancement ability than smooth ones because of the stronger electromagnetic field. Various kinds of hotspot-rich NPs with rough surfaces have been elaborately designed, such as gold nanoflowers via one-pot asymmetric growth, ${ }^{17}$ gold nanostars via seed-mediated preparation, ${ }^{18}$ gold nanocorn obtained from polyhistidine coating and seed growth, ${ }^{19}$ and core-satellite NPs prepared by self-assembly. ${ }^{20,21}$ Although silver coating is beneficial for signal enhancement of $\mathrm{Au} @ \mathrm{Ag}$ NPs, some problems related to the surface topography variation exist. Silver randomly grew on the cores without any site and density regulation, which tended to smooth the surface of core NPs, merging the tips, holes, or junctions of the core NPs (especially for nanoflowers ${ }^{22}$ and nanostars ${ }^{10}$ ). Thus, the resultant bimetallic NPs were usually spherical and smooth and lost the intrinsic hotspots of the cores. Additionally, the morphological changes resulted in a significant blue shift of localized surface plasmon resonance (LSPR) to the position far from the laser wavelength (usually 632.8 or $780 \mathrm{~nm}$ ), which was disadvantageous for the generation of ultrasensitive surface-enhanced resonant Raman scattering (SERRS) signals.

Several works endeavored to synthesize asymmetric bimetallic NPs to combine the advantages of both material and surface morphology, by controlling silver NP growing density and direction on gold cores. ${ }^{23-25}$ However, these NPs were of simple two-dimensional structures with the two (or few) metal spheres directly connected and displayed uneven Ag NP distribution (Figure S1). The design of a threedimensional (3D) structure with discontinuous and uniform Ag nanotopography, especially in synthesizing Au@Ag IS tags became an important direction to improve SERS performance of these tags. This task is still challenging and such bimetallic NP structures have never been reported to the best of our knowledge.

Recently, we designed waxberry-like Au NPs, which had vertically oriented gold nanorod (AuNR) arrays deposited on a large gold nanosphere (AuNS). ${ }^{26}$ Herein, we developed 3D $\mathrm{Au} @ \mathrm{Ag}$ NPs for the first time by selecting waxberry-like $\mathrm{Au}$ $\mathrm{NPs}$ as the cores. As shown in Figure 1A, the proposed synthesis protocol made the full use of the anisotropic effect and spatial distribution of the AuNR arrays. The thiol-ligands (serving as gold surface blocking agents) preferred to cap on flat AuNR sides and formed a dense molecular layer. In contrast, the capping density at the tips was much lower because of the large curvature, supplying bare $\mathrm{Au}$ sites at the tips. After the addition of L-ascorbic acid (AA) and $\mathrm{AgNO}_{3}, \mathrm{Ag}$ was forced to selectively grow on the bare sites, forming uniform AuNS-AuNR-AgNP 3D nanostructures with matchlike arrays over the cores.

There were not only high-density homo- (AgNP-AgNP) hotspots on the surface but also AuNR-bridged hetero(AuNS-Ag NP) hotspots within the inner gaps and holes of the single 3D NPs. The 3D hotspots endowed the NP with extraordinary SERS enhancement ability that was 30 times higher than conventional Au@Ag NPs with a smooth surface, 
realizing signal detection from a single NP. The morphology, uniformity, and size distribution of the products were excellent. More interestingly, "blank" or "IS"-coded NPs with multiplex Raman signatures can be developed by simply changing the thiol-ligands, which illustrated the synthesis generality and versatility. The multifunctionality of the NPs has been demonstrated in several important fields, such as bioimaging and photothermal therapy, as well as pollutant identification and quantitative analysis via a ratiometric strategy.

\section{EXPERIMENTAL SECTION}

Materials. 4-Nitrothiophenol (NT), cetyltrimethylammonium bromide (CTAB), 3,3'-diethylthiatricarbocyanine iodide (DTTC), cetyltrimethylammonium chlorine, 4-aminothiophenol (NAT), 1,4benzenedithiol (BDT), 4-fluorothiophenol (FT), $\beta$-mercaptoethylamine (MEA), 4-mercaptobenzoic acid (MBA), and mercaptopyridine (MPY) were purchased from Sigma-Aldrich. Sodium borohydride $\left(\mathrm{NaBH}_{4}\right)$, chloroauric acid $\left(\mathrm{HAuCl}_{4}\right)$, crystalviolet $(\mathrm{CV})$, rhodamine6G $(\mathrm{R} 6 \mathrm{G})$, sodium hydroxide $(\mathrm{NaOH})$, AA, ethyl alcohol absolute, ammonium hydroxide $\left(\mathrm{NH}_{3} \cdot \mathrm{H}_{2} \mathrm{O}\right)$, silver nitrate $\left(\mathrm{AgNO}_{3}\right)$, and tetraethoxysilane (TEOS) were obtained from Sinopharm Chemical Reagent Co. Ltd. Thiram (97\%) was obtained from Macklin. Cyanine 7 monoacid (Cy7) and (E)-2-((4-(phenylethynyl)benzylidene)amino)ethanethiol (PBAT) were synthesized in our laboratory. Lipoid E80 was purchased from Lipoid GmbH. Penicillin-streptomycin solution, Dulbecco's modified Eagle's medium, trypsin-ethylenediaminetetraacetate solution, and fetal bovine serum were obtained from Thermo Scientific. We use deionized water in all the experiments.

Characterization. SERS spectra were obtained on a DXR Raman microscope (Thermo Scientific, USA) with a $10 \times$ microscope objective for sample solution measurement and a $50 \times$ microscope objective for cell mapping. UV/vis/near infrared (NIR) absorption spectra were performed by a Thermo Scientific NanoDrop 2000/ 2000C spectrophotometer. Dark-field was obtained by a BD microscope (NIKON, Japan). Zetasizer Nano ZS90 (Malvern Instruments, Malvern, U.K.) was used to record dynamic light scattering results. Scanning electron microscopy (SEM) images were acquired on an S-4800 field-emission scanning electron microscope (Hitachi, Japan). The way to statistical analysis of NP size distribution in SEM images was by using Nano Measure software. The thermal imager was obtained by Testo 865 (Germany).

Synthesis of 3D Au@Ag NPs. The waxberry-like Au core was synthesized according to our reported work. The detailed synthesis procedure can be found in the Supporting Information. The synthesized waxberry-like $\mathrm{Au}$ core $(1.5 \mathrm{~mL})$ was first added to the thiol-ligands such as NT, NAT, BDT, MBA, FT, PBAT, MPY, and MEA $\left(10^{-3} \mathrm{M}, 15 \mu \mathrm{L}\right)$. After placing at room temperature for $6 \mathrm{~h}$, the solution was centrifuged for $10 \mathrm{~min}$ with $3000 \mathrm{rpm}$ and the pellet was resuspended in CTAB $(1.5 \mathrm{~mL}, 1 \mathrm{mM})$. Then, $\mathrm{AgNO}_{3}(0.01 \mathrm{M}, 100$ $\mu \mathrm{L})$, AA $(0.01 \mathrm{M}, 100 \mu \mathrm{L})$, and $\mathrm{NH}_{3} \cdot \mathrm{H}_{2} \mathrm{O}(2.8 \%, 20 \mu \mathrm{L})$ were added to $1.5 \mathrm{~mL}$ of the prepared $\mathrm{CTAB}$ solution under gentle stirring at room temperature. The initially green colored mixture solution turned to gray during $1 \mathrm{~min}$, revealing the formation of 3D Au@Ag NPs. The preparation of core-shell NPs was similar to that of 3D Au@Ag NPs except not using any thiol-ligands or using the CV, R6G, and DTTC as the thiol-ligands. For obtaining various amounts of $\mathrm{Ag}$ coating with 3D Au@Ag NPs or core-shell NPs, the corresponding volume of AA (0.01 M), $\mathrm{AgNO}_{3}(0.01 \mathrm{M})$, and $\mathrm{NH}_{3} \cdot \mathrm{H}_{2} \mathrm{O}(2.8 \%)$ should be changed, and the volume ratio among $\mathrm{AA}(0.01 \mathrm{M}), \mathrm{AgNO}_{3}(0.01$ $\mathrm{M})$, and $\mathrm{NH}_{3} \cdot \mathrm{H}_{2} \mathrm{O}(2.8 \%)$ was kept at 5:5:1. In order to investigate the influence of different thiol-ligand concentrations on the synthesis of NPs, different concentrations of MEA were added to the waxberrylike Au core, respectively, and the synthesis procedure of 3D Au@Ag NPs was similar to the above.

Single Particle SERS Detection. Cy7 $\left(10^{-4} \mathrm{M}, 10 \mu \mathrm{L}\right)$ was added into $200 \mu \mathrm{L}$ of 3D Au@Ag NPs. After placing the solution at room temperature for $0.5 \mathrm{~h}$, it was centrifuged for $10 \mathrm{~min}$ with 2500 rpm and diluted 200 times. $10 \mu \mathrm{L}$ of this diluted solution was dropped on a cleaned indium tin oxide (ITO) and the surrounding area of 3D $\mathrm{Au} @ \mathrm{Ag}$ was marked with carbon. It was dried under natural conditions to realize the observation of a single particle in a darkfield. Then, the Raman intensity detection can be obtained with a 780 $\mathrm{nm}$ laser wavelength. The exposure time was $5 \mathrm{~s}$ and the laser power was $100 \mathrm{~mW}$.

Preparation of the PBAT Encoded 3D Au@Ag NPs for SERS Quantitation of Thiram. The encoded 3D Au@Ag NPs were first synthesized by using PBAT as the thiol-ligand. Afterward, $600 \mu \mathrm{L}$ of the encoded 3D Au@Ag NPs were centrifuged for 10 min with 2500 $\mathrm{rpm}$ and redispersed in $100 \mu \mathrm{L}$ of different concentrations of thiram solution $\left(10^{-4}\right.$ to $\left.10^{-7} \mathrm{M}\right)$, which was directly used for SERS detection at a $780 \mathrm{~nm}$ laser wavelength with $5 \mathrm{~s}$ of exposure time. The laser power was $100 \mathrm{~mW}$. The linear relationship between the Raman intensity ratio $\left(I_{\text {thiram }} / I_{\mathrm{PBAT}}\right)$ or Raman intensity $\left(I_{\text {thiram }}\right)$ and the concentration of thiram was calculated to evaluate the calibration capability from thiol-ligands which was served as an IS. The lake water sample was spiked with $10^{-6} \mathrm{M}$ of thiram. Then, $600 \mu \mathrm{L}$ of PBAT-encoded 3D Au@Ag NPs was centrifuged for 10 min with $2500 \mathrm{rpm}$ and redispersed in $100 \mu \mathrm{L}$ of lake water for SERS detection.

Preparation of Silica-Coated 3D Au@Ag NP-Based SERS Tags. First, DTTC $\left(10^{-4} \mathrm{M}, 20 \mu \mathrm{L}\right)$ was added to $2 \mathrm{~mL}$ of 3D Au@ Ag NPs by using MEA as a thiol-ligand. Then, the solution was kept away at room temperature for $0.5 \mathrm{~h}$ and subsequently centrifuged at $2500 \mathrm{rpm}$ for $10 \mathrm{~min}$ to obtain DTTC-labeled 3D Au@Ag NPs. Then, silica-coated 3D Au@Ag NP-based SERS tags were acquired based on a reported method with minor modification. Briefly, $2 \mathrm{~mL}$ of the above 3D Au@Ag NP-based SERS tags were centrifuged for 10 $\mathrm{min}$ at $2500 \mathrm{rpm}$ and the pellets were redispersed in CTAB $(5 \mathrm{mM}, 1$ $\mathrm{mL})$, followed by addition of $\mathrm{NaOH}(0.1 \mathrm{M}, 20 \mu \mathrm{L})$. Then, $8 \mu \mathrm{L}$ of TEOS $(20 \%)$ was added to the previous mixture every $0.5 \mathrm{~h}$ and it was repeated three times. After gently stirring at room temperature for 3 days. The silica-coated 3D Au@Ag NP-based SERS tags were finally rinsed with ethanol for three times and deionized water for one time.

Cell Imaging. First, glass coverslips were put into a 24 -well plate with $500 \mu \mathrm{L}$ of A549 cells $\left(2 \times 10^{4}\right)$. Then, the cells were allowed to seed onto the glass coverslips after incubation for $12 \mathrm{~h}$. Silica-coated 3D Au@Ag NP-based SERS tags $(350 \mu \mathrm{g} / \mathrm{mL}, 150 \mu \mathrm{L})$ were added into $500 \mu \mathrm{L}$ of the cell medium. After $3 \mathrm{~h}$, phosphate-buffered saline (PBS) was used to repeatedly wash the cell monolayer on the coverslip for removing the remaining particles that had not entered into the cells. Finally, the cover-slip was sealed with a glass microscope slide for SERS mapping via DXR Raman microscopy with a step size of 3 $\mu \mathrm{m}$ at a $780 \mathrm{~nm}$ laser wavelength. The exposure time was $5 \mathrm{~s}$ and the laser power was $4 \mathrm{~mW}$.

In Vivo Imaging. $200 \mu \mathrm{L}$ of silica-coated 3D Au@Ag NP-based NIR SERRS tags $\left(400 \mu \mathrm{g} / \mathrm{mL}\right.$, containing $5 \times 10^{-6} \mathrm{M}$ of cy 7$)$ were injected into a tumor-bearing mouse (xenograft tumor of A549 human lung cancer cells) intravenously via the tail vein. After $1.5 \mathrm{~h}$ of injection, SERS spectra at tumor sites, blank skin, the tail, and the liver were in situ measured at a $780 \mathrm{~nm}$ laser wavelength with $5 \mathrm{~s}$ of exposure time. The laser power was $140 \mathrm{~mW}$.

In Vivo Temperature Maps of the Mouse with 3D Au@Ag NPs. The 3D Au@Ag NPs $(300 \mu \mathrm{g} / \mathrm{mL}, 120 \mu \mathrm{L})$ and PBS were injected into the subcutaneous tissue of the nude mouse, respectively. Then the injection sites were irradiated with a $785 \mathrm{~nm}$ laser for $110 \mathrm{~s}$. The power intensity was $3.6 \mathrm{~W} / \mathrm{cm}^{2}$. A thermal imager (Testo 865 ) was used to monitor the temperature of the injected sites every $10 \mathrm{~s}$.

\section{RESULTS AND DISCUSSION}

Synthesis of 3D Au@Ag NPs. Figure 1B shows the SEM image of the waxberry-like Au NP cores. Vertically oriented AuNR arrays (around $5 \times 25 \mathrm{~nm}$ ) can be easily observed from an elaborately selected NP with an open pore on the core surface. The diameter of the waxberry-like NP was approximately $110 \mathrm{~nm}$. After thiol-ligand (MEA) capping and $\mathrm{Ag}$ growth, the surface changed to a match-like array with a large number of AgNPs over the tips of the AuNRs (Figure 
1C). The size, the surface density of the AgNPs, and the optical properties of the Au@Ag NPs can be tunable via changing the amount of $\mathrm{AgNO}_{3}$ (Figure S2). In the optimized amount of $\mathrm{AgNO}_{3}(10 \mathrm{mM}, 100 \mu \mathrm{L})$, the diameter of AgNPs was in a narrow range of $24-32 \mathrm{~nm}$, and the gaps among them were less than $10 \mathrm{~nm}$. The 3D Au@Ag NPs also had uniform morphology and narrow size distribution in the range from 140 to $170 \mathrm{~nm}$ (Figure 1D). The silver growth induced an abrupt color change from green of the waxberry-like core NPs to gray of the 3D Au@Ag NPs. The LSPR wavelength of waxberry-like core NPs red-shifted about $120 \mathrm{~nm}$ owing to the strong coupling effect between the core and external Ag NPs. A new peak appeared at $425 \mathrm{~nm}$, which can be assigned to the contribution of the AgNPs (Figure 1E). The zeta potential of the 3D Au@Ag NPs was $24.2 \mathrm{mV}$, indicating the colloidal stability of the solution. Taking advantage of the hotspots of the 3DAu@Ag NPs, the SERS ability enhanced about 50 times higher than the waxberry-like Au cores (Figure 1F).

The unique structure of the waxberry-like core NPs provides a premise for controllable silver growth (including growth site, direction, and density), relying on the foundation of 3D Au@ Ag NP development. First, the anisotropic property of AuNRs caused the thiol molecule agent to selectively block the sides, $^{27-29}$ which regulated the silver growth sites and directions. Silver seeds were forced to form at the tips of AuNRs and further grew upward, preventing fast fusion. The blocking extent of the AuNR surface determined silver growth sites and final morphology. ${ }^{23-25,30}$ If the side of the AuNRs was not effectively blocked, for instance, without any capping agent (Figure S3) or with insufficient thiol molecule capping agents (MEA concentration lower than $10^{-5} \mathrm{M}$, as shown in Figures 2 and S4), or using nonthiol molecules like CV, R6G,

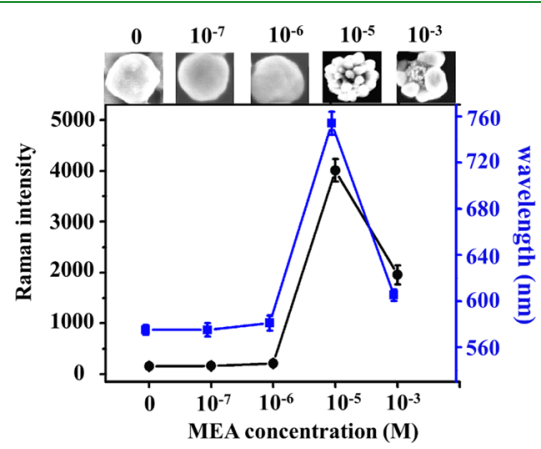

Figure 2. Effect of MEA concentration on the morphology, SERS enhancement ability, and LSPR peak position of the resultant $\mathrm{Au} @ \mathrm{Ag}$ NPs. DTTC $\left(10^{-6} \mathrm{M}\right)$ was used for SERS measurement. The data were presented as the mean intensity of the $1247 \mathrm{~cm}^{-1}$ peak with standard deviation $(n=3)$.

or DTTC (Figure S5), silver evenly grew around each AuNR and finally merged the whole AuNR arrays. As a result, only conventional bimetallic core-shell NPs with a smooth and continuous surface can be obtained. On the contrary, when the concentration of thiol molecules was too high $\left(10^{-3} \mathrm{M}\right)$, most tips were also blocked and the number of growth sites on AuNRs sharply decreased, resulting in the formation of a few inhomogeneous big silver islands over the AuNRs (Figures 2 and $\mathrm{S} 4 \mathrm{C}) .{ }^{31}$

Second, the vertically orientated AuNRs offered segregated silver growth sites, which was also crucial for the formation of this discontinuous surface morphology. If MEA-capped AuNSs were used as the core, irregular silver "islands" formed on the core surface because of the random growth sites and uncontrollable silver fusion during growth (Figure 3B,C).

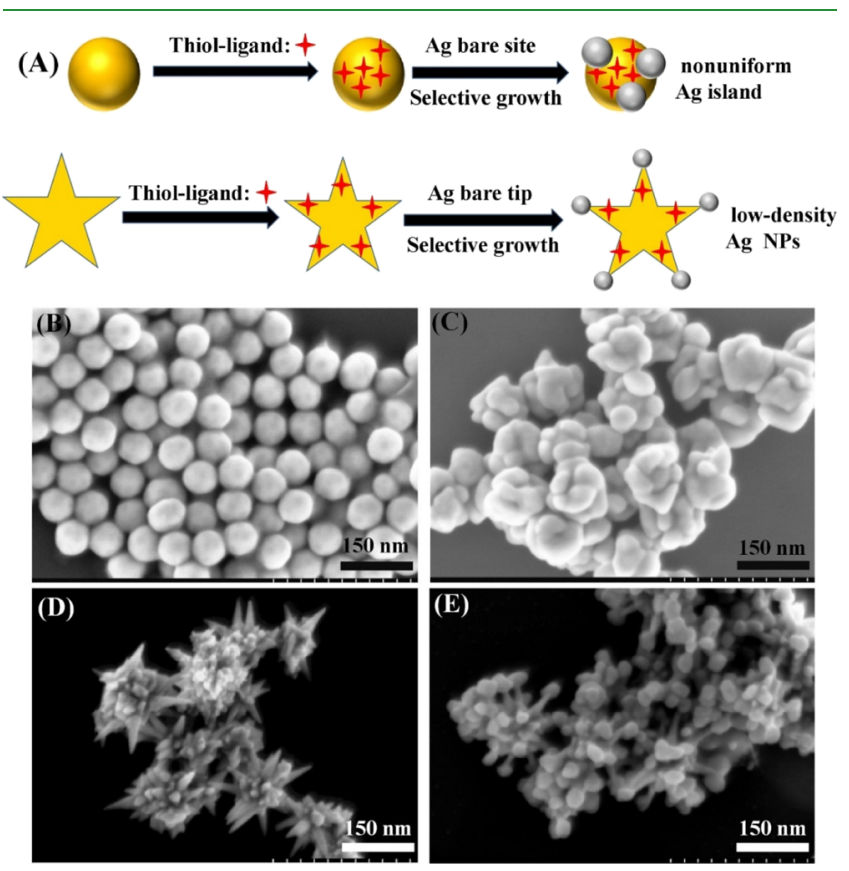

Figure 3. (A) Schematic illustration of the synthesis of Au@Ag NPs with MEA-capped AuNSs and Au nanostars as the core. SEM images of the MEA $\left(10^{-5} \mathrm{M}\right)$-capped AuNS core (B) and Au nanostar core (D) and the resultant Au@Ag NPs (C,E).

Multibranched-Au nanostars were also tested as the core. ${ }^{32}$ Despite the fact that $\mathrm{Ag}$ could selectively grow on the tips (Figure 3D,E), the size and uniformity of the resultant bimetallic NPs were unsatisfactory. More importantly, the Ag NPs were far from each other, which made it hard to generate dense built-in "hotspots" because of the limited number of tips of a $\mathrm{Au}$ nanostar.

On the surface of a single 3D Au@Ag NP, there were not only much hotspot to provide the strong electromagnetic field for SERS enhancement, but also a large surface area for analytes binding. Besides, it had an ideal NIR LSPR peak position $(760 \mathrm{~nm})$ that well matched with the wavelength of irradiation laser $(780 \mathrm{~nm})$, leading to the SERRS effect and improving the enhancement ability. All these benefits were attributed to the 3D Au@Ag NP extraordinary enhancement ability, which was around 30 times higher than that of the smooth Au@Ag core-shell NPs (Figure 2). A 632.8 nm laser was also used for comparison. As shown in Figure S6, the intensity of 3D NPs was 23 times higher than that of the smoothAu@Ag core-shell NPs. The value was less than that obtained from a $780 \mathrm{~nm}$ laser mainly because of the decreased resonance effect. Moreover, the analytical enhancement factor $(\mathrm{AEF})$ of the 3D Au@Ag NPs reached $3.4 \times 10^{6}$ at $780 \mathrm{~nm}$ excitation (see Supporting Information), which was $10-100$ times greater than the values from the reported bimetallic Au@ Ag metal NPs. ${ }^{33,34}$

Taking advantage of the high-sensitivity of 3D Au@Ag NPs, single particle SERS measurement can be realized. To the best use of SERRS, the cy7-labeled NPs were dropped on an ITO glass slide. The single NP distribution was investigated by SEM and dark-field images (Figure 4A,B). Figure 4C depicts SERS 

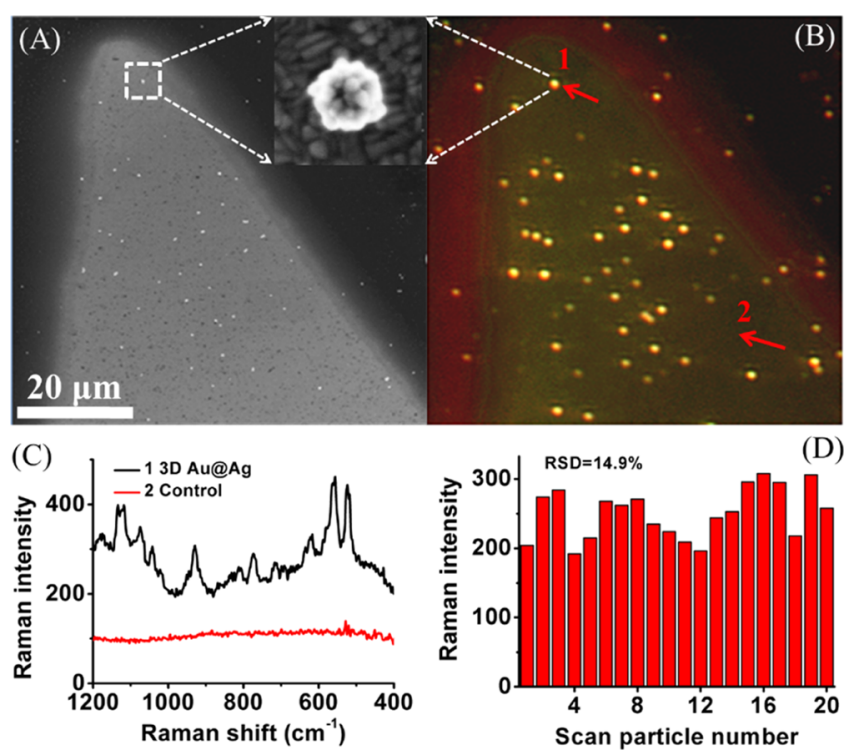

Figure 4. SEM image (A) and the corresponding dark-field image (B) of 3D Au@Ag NPs spread on the ITO glass. The inset illustrates a single NP for SERS measurement. (C) SERS spectra of the single particle and a blank site. (D) SERS intensity distribution histogram of the randomly chosen 20 particles at $530 \mathrm{~cm}^{-1}$.

spectra of a single particle and a blank site (ITO), exhibiting strong and characteristic Raman peaks of cy7 from the single 3D Au@Ag NP, while the blank ITO had no signal. SERS spectra of twenty randomly selected single NPs were recorded from the dark-field images (Figure S7), and the RSD of the peak intensity at $530 \mathrm{~cm}^{-1}$ was calculated to be $14.9 \%$, illustrating the signal uniformity among the single NPs (Figure 4D). ${ }^{35,36}$

3D Au@Ag NPs with Various SERS Signatures. Multiplexing Raman signatures can be achieved by simply changing thiol-ligands. Apart from the abovementioned SERS inactive MEA, the capping agent has been successfully extended to a large set of SERS active thiol molecules. Figure 5 shows SEM images and SERS spectra of 3D NPs prepared with 8 representative thiol-ligands. The NPs have similar 3D structure, and SERS intensity of IS thiol molecules (NT) was about 26 times higher than that of the waxberry-like Au core (Figure S8). Excitingly, these spectrum signatures can be divided into two groups, that is, MEA-based "blank" NPs showing a clean signal background, and SERS active thiol molecule-based NPs producing strong "IS" signals because of the encapsulation of capping agents between the metals. More interestingly, four characteristic peaks of NT $\left(851 \mathrm{~cm}^{-1}\right)$, NAT $\left(1594 \mathrm{~cm}^{-1}\right)$, BDT $\left(735 \mathrm{~cm}^{-1}\right)$, and FT $\left(582 \mathrm{~cm}^{-1}\right)$ can be also separately observed (Figure 5), making these IS tags suitable for multiplex detection. Recently, the interests in IS SERS tags has been increasing because of the stable IS signal and analyte-sensing ability of the open surface. ${ }^{37,38}$ Herein, we first proposed 3D IS tag groups via this simple synthesis method. These tags have great potential for multiplex labeling and quantitative detection of analytes via a ratiometric strategy. Besides, multiplex detection capabilities of "blank" NPs can be illustrated by detecting and distinguishing SERS characteristic peaks of cocktail solutions composed of different kinds of representative Raman reporters (Figure S9).

"Blank" NP-Based SERRS Tags for Bioimaging. The different signal signatures of "blank" and "IS" NPs implied the

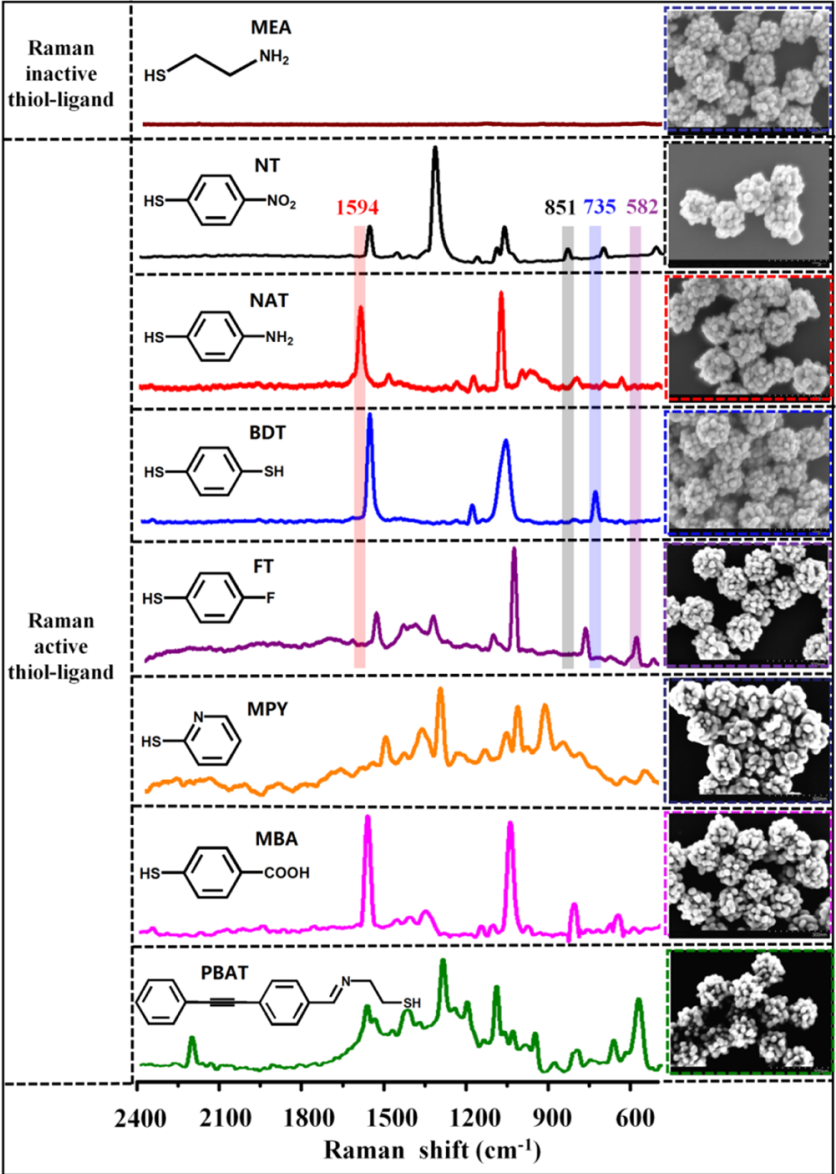

Figure 5. SERS spectra and SEM images of 3D Au@Ag NPs prepared with different thiol-ligands. Four characteristic peaks of NT, NAT, $\mathrm{BDT}$, and FT can be separated, making these IS tags useful for multiplex detection.

application versatility. The clean background of "blank" NPs demonstrated their sensing and identifying ability toward various environmentally relevant analytes, such as malachite green, phosphorus triphenyl, TNT, melamine, and thiram (Figure S10). The NPs showed satisfactory long-term stability, and the structure and sensing ability were unchanged after 30 day storage as well (Figure S11). Another application was the synthesis of ultrasensitive NIR SERRS tags for bioimaging (Figure 6A). To increase the biocompatibility of the "blank" NPs, silica-coated tags were prepared (Figure S12) and then incubated with A549 cells. ${ }^{39,40}$ As shown in Figure 6B, bright yellow dots can be observed in the cells after incubation for 3 $\mathrm{h}$, and the Raman signal of the DTTC reporters can be clearly detected. Figure 6C,D show the SERS mapping image of the cells, which proved the living cell labeling ability of the tags.

To demonstrate the in vivo imaging potential of NIR SERRS tags, a proof-of-concept experiment was performed in a tumorbearing nude mouse. Cy7-encoded tags with silica coatings were injected into the mouse through the tail vein (Figure 6E). After circulation for $1.5 \mathrm{~h}$, strong Raman signals can be in situ detected from blood and the liver. Pronounced Raman peaks also showed at the tumor site, implying the tags can passively penetrate the tumor and hold a promise for tumor diagnosis (Figure 6F). The 3D nanotopography also endowed the tags with enhanced NIR light absorption property, thus obtaining satisfactory photothermal ability. As shown in Figure 6G,H, the 

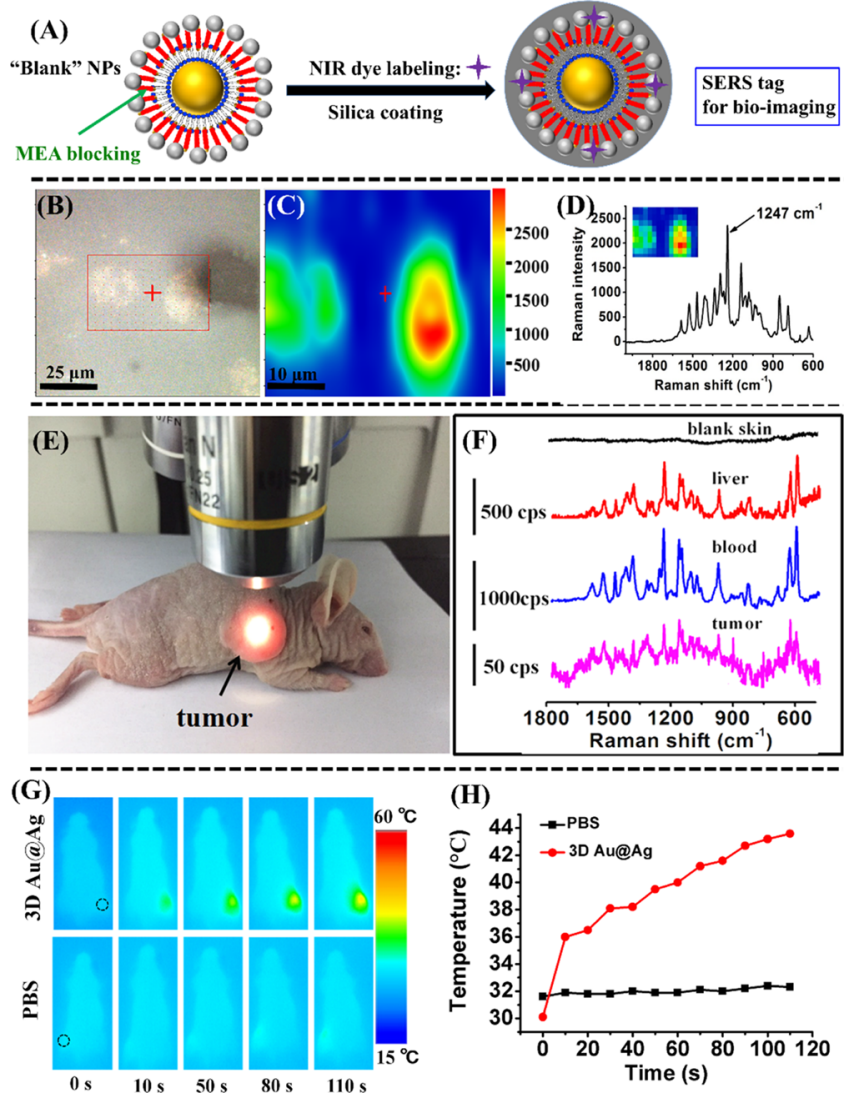

Figure 6. (A) Schematic preparation of the "blank" NP-based SERRS tags for bioimaging. (B) Dark-field image and (C) SERS mapping image of living cells labeled with the tags encoded by a DTTC Raman reporter. The concentration of the tag was $80 \mu \mathrm{g} / \mathrm{mL}$. The mapping is drawn from the intensity of the $1247 \mathrm{~cm}^{-1}$ peak. (D) Characteristic DTTC signal extracted from the mapping image of the cells. (E) In vivo SERS detection of an A549 tumor-bearing mouse intravenously injected with cy7-labeled NIR SERRS tags. (F) SERS spectra in situ detected from the liver, blood, and tumor of the mouse $1.5 \mathrm{~h}$ after injection. (G) Thermal imaging results of a nude mouse subcutaneously injected with the tags $(300 \mu \mathrm{g} / \mathrm{mL})$ and PBS solution at the legs with different laser irradiation $\left(785 \mathrm{~nm}, 3.6 \mathrm{~W} / \mathrm{cm}^{2}\right)$ times. $(\mathrm{H})$ Temperature evolution of injected sites.

temperature of the NP-injection site of a nude mouse dramatically increased from 31 to $43{ }^{\circ} \mathrm{C}$ within $110 \mathrm{~s}$ laser irradiation $\left(785 \mathrm{~nm}, 3.6 \mathrm{~W} / \mathrm{cm}^{2}\right)$. The versatility of the tags offered the platform for the development of multifunctional theranostic agents.

"IS" NPs for Ratiometic Detection of Pesticides. The feasibility of "IS" NPs for an application of quantitative detection was tested by using thiram as a model analyte (Figure 7A). Alkynyl-containing PBAT $^{41}$ was synthesized as a thiol-ligand to prepare IS NPs because it can generate a characteristic Raman peak in the Raman-silence region (around $2220 \mathrm{~cm}^{-1}$ ). This peak would not overlay with most analytes' peaks and can be easily distinguished as an IS signal. $^{42-45}$ Different concentrations of thiram were added to the IS NP solution, followed by SERS spectra measurements. As indicated in Figure 7B, characteristic peaks of both molecules can be detected and differentiated (PBAT 2220 $\mathrm{cm}^{-1}$; thiram $\left.1380 \mathrm{~cm}^{-1}\right)$. The raw data directly from SERS intensity of thiram $\left(I_{1380}\right)$ gave a poor linear relationship $\left(R^{2}=\right.$ 0.821 ) because of possible inhomogeneous aggregation of NPs

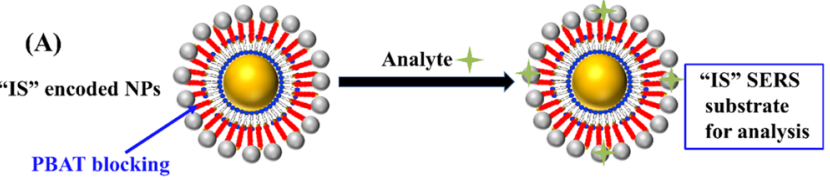

(B)

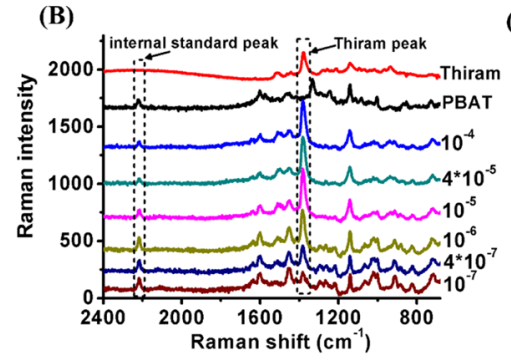

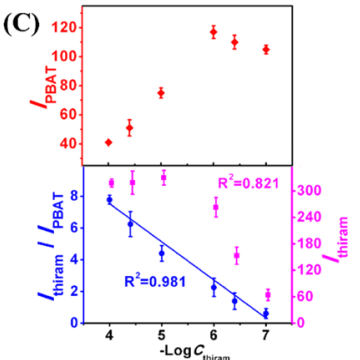

Figure 7. (A) Schematic of "IS" NPs for pesticide quantitative detection. (B) SERS spectra of thiram (red line, obtained from "blank" 3D NPs), IS tag (black line, obtained from PBAT using a thiol-ligand), and IS tag mixed with different concentrations of thiram. The IS peak of PBAT $\left(2220 \mathrm{~cm}^{-1}\right)$ and the thiram peak $(1380$ $\mathrm{cm}^{-1}$ ) for ratiometric analysis are indicated by dashed boxes. (C) Plots of the SERS intensities of the IS PBAT (red dot), thiram (purple square), and relative intensities (blue dot) at different concentrations of thiram.

and the subsequent precipitation of large aggregates, as reflected by the reduced SERS intensity of IS with the increasing concentration of thiram. ${ }^{13}$ The linearity of the curve was effectively improved $\left(R^{2}=0.981\right)$ by normalizing the data to the IS intensities of PBAT $\left(I_{1380} / I_{2220}\right)$ in a wide concentration range of thiram from $10^{-7}$ to $10^{-4} \mathrm{M}$ (Figure 7C). Apparently, the IS signal calibrated the NP state and measurement variations and played a vital role in the quantitative SERS analysis. $10^{-7} \mathrm{M}$ of thiram can be easily detected, which was comparable to the reported values obtained from hotspot-rich metal NP colloid-based SERS detection. $^{46}$ The possibility of practical detection was demonstrated on a spiked lake water sample containing a trace amount of thiram $\left(10^{-6} \mathrm{M}\right)$. Satisfactory recovery (92.3\%) was obtained, suggesting great potential of "IS" NPs for environmental pollutant detection (Figure S13).

\section{CONCLUSIONS}

In summary, we developed novel 3D Au@Ag NPs with uniform morphology, ultrasensitivity, and application versatility. The waxberry-like Au core structure and selective surface blocking played a crucial role in the silver growth site, direction, and density. Compared with traditional Au@Ag NPs with a smooth silver shell, our 3D NPs showed many adjacent but discontinuous silver NPs over the cores. This was helpful to construct stereoscopic hotspots, endowing the SERS detection sensitivity at a single particle level. This protocol was general and could produce NPs encoded with two types of SERS signatures (blank and multiplex IS signals) by simply changing the thiol-ligands. Proof-of-concept applications have been successfully demonstrated in the field of in vivo bioimaging and pollutant quantitative analysis. The satisfactory optical properties and versatility would make the tags ideal candidates in the wide SERS-related research fields. 


\section{ASSOCIATED CONTENT}

\section{S Supporting Information}

The Supporting Information is available free of charge at https://pubs.acs.org/doi/10.1021/acsami.9b18292.

Schematic illustration of the evolution of $\mathrm{Au} @ \mathrm{Ag}$ bimetallic NPs, SEM images and optical characteristics of different 3D Au@Ag NPs, schematic illustration of the formation mechanism of core-shell Au@Ag NPs, SEM images of 3DAu@Ag NPs prepared with different concentrations of MEA, SEM images of smooth Au@Ag NPs, SERS spectra of DTTC upon the $632.8 \mathrm{~nm}$ laser wavelength, dark-field images of twenty randomly selected single NPs, SERS spectra of NT from 3D Au@Ag NPs and core-shell Au@Ag NPs, SERS spectra of three reporters, SERS signals of various analytes, stability data of 3D Au@Ag NPs, SEM image and cell viability of silica-coated SERS tags, and SERS spectra of 3D Au@Ag NPs used for the detection of lake water (PDF)

\section{AUTHOR INFORMATION}

\section{Corresponding Authors}

*Email: yqwang@yic.ac.cn (Y.W.).

*Email: lxchen@yic.ac.cn. Phone: +86 535 2109130. Fax: +86 5352109130 (L.C.).

\section{ORCID $\odot$}

Lingxin Chen: 0000-0002-3764-3515

\section{Notes}

The authors declare no competing financial interest.

\section{ACKNOWLEDGMENTS}

Financial support from the National Natural Science Foundation of China (81573393 and 21976209), the Science and Technology Development Plan of Shandong Province of China (2019GSF108047), the Youth Innovation Promotion Association CAS (2017256), the Instrument Developing Project of the Chinese Academy of Sciences (YZ201662), the Technical Innovation Project of Instrument Function Development of the Chinese Academy of Sciences, and Taishan Scholar Project Special Funding (no. ts20190962) is gratefully acknowledged.

\section{REFERENCES}

(1) Lin, M.; Wang, Y.; Sun, X.; Wang, W.; Chen, L. "Elastic" Property of Mesoporous Silica Shell: for Dynamic Surface Enhanced Raman Scattering Ability Monitoring of Growing Noble Metal Nanostructures via a Simplified Spatially Confined Growth Method. ACS Appl. Mater. Interfaces 2015, 7, 7516-7525.

(2) Su, X.; Wang, Y.; Wang, W.; Sun, K.; Chen, L. Phospholipid Encapsulated AuNR@Ag/Au Nanosphere SERS Tags with Environmental Stimulus Responsive Signal Property. ACS Appl. Mater. Interfaces 2016, 8, 10201-10211.

(3) Wang, Y.; Yan, B.; Chen, L. SERS Tags: Novel Optical Nanoprobes for Bioanalysis. Chem. Rev. 2013, 113, 1391-1428.

(4) Han, G.; Liu, R.; Han, M.-Y.; Jiang, C.; Wang, J.; Du, S.; Liu, B.; Zhang, Z. Label-Free Surface-Enhanced Raman Scattering Imaging to Monitor the Metabolism of Antitumor Drug 6-Mercaptopurine in Living cells. Anal. Chem. 2014, 86, 11503-11507.

(5) Su, J.; Wang, D.; Nörbel, L.; Shen, J.; Zhao, Z.; Dou, Y.; Peng, T.; Shi, J.; Mathur, S.; Fan, C.; Song, S. Multicolor Gold-Silver NanoMushrooms as Ready-to-Use SERS Probes for Ultrasensitive and Multiplex DNA/miRNA Detection. Anal. Chem. 2017, 89, 25312538.
(6) Zeng, Y.; Pei, J.-J.; Wang, L.-H.; Shen, A.-G.; Hu, J.-M. A Sensitive Sequential 'on/off SERS Assay for Heparin with Wider Detection Window and Higher Reliability Based on the Reversed Surface Charge Changes of Functionalized Au@Ag Nanoparticles. Biosens. Bioelectron. 2015, 66, 55-61.

(7) Zhou, Y.; Lee, C.; Zhang, J.; Zhang, P. Engineering Versatile SERS-Active Nanoparticles by Embedding Reporters between AuCore/Ag-Shell through Layer-by-Layer Deposited Polyelectrolytes. J. Mater. Chem. C 2013, 1, 3695-3699.

(8) Wang, Y.; Wang, Y.; Wang, W.; Sun, K.; Chen, L. ReporterEmbedded SERS Tags from Gold Nanorod Seeds: Selective Immobilization of Reporter Molecules at the Tip of Nanorods. ACS Appl. Mater. Interfaces 2016, 8, 28105-28115.

(9) Khlebtsov, B.; Khanadeev, V.; Khlebtsov, N. Surface-Enhanced Raman Scattering Inside Au@Ag Core/Shell Nanorods. Nano Res. 2016, 9, 2303-2318.

(10) Fales, A. M.; Vo-Dinh, T. Silver Embedded Nanostars for SERS with Internal Reference (SENSIR). J. Mater. Chem. C 2015, 3, 73197324.

(11) Li, J.; Zhu, Z.; Zhu, B.; Ma, Y.; Lin, B.; Liu, R.; Song, Y.; Lin, H.; Tu, S.; Yang, C. SERS-active Plasmonic Nanoparticles with Ultrasmall Interior Nanogap for Multiplex Quantitative Detection and Cancer Cell Imaging. Anal. Chem. 2016, 88, 7828-7836.

(12) Jiang, X.; Tan, Z.; Lin, L.; He, J.; He, C.; Thackray, B. D.; Zhang, Y.; Ye, J. Surface-Enhanced Raman Nanoprobes with Embedded Standards for Quantitative Cholesterol Detection. Small Methods 2018, 2, 1800182.

(13) Shen, W.; Lin, X.; Jiang, C.; Li, C.; Lin, H.; Huang, J.; Wang, S.; Liu, G.; Yan, X.; Zhong, Q.; Ren, B. Reliable Quantitative SERS Analysis Facilitated by Core-Shell Nanoparticles with Embedded Internal Standards. Angew. Chem., Int. Ed. 2015, 54, 7308-7312.

(14) Li, Y.; Shi, Q.; Zhang, P.; Xiahou, Y.; Li, S.; Wang, D.; Xia, H. Empirical Structural Design of Core@Shell Au@Ag Nanoparticles for SERS Applications. J. Mater. Chem. C 2016, 4, 6649-6656.

(15) Shen, J.; Su, J.; Yan, J.; Zhao, B.; Wang, D.; Wang, S.; Li, K.; Liu, M.; He, Y.; Mathur, S.; Fan, C.; Song, S. Bimetallic NanoMushrooms with DNA-Mediated Interior Nanogaps for HighEfficiency SERS Signal Amplification. Nano Res. 2014, 8, 731-742.

(16) Jiang, T.; Wang, X.; Zhou, J. The Synthesis of Four-Layer GoldSilver-Polymer-Silver Core-Shell Nanomushroom with Inbuilt Raman Molecule for Surface-Enhanced Raman Scattering. Appl. Surf. Sci. 2017, 426, 965-971.

(17) Xie, J.; Zhang, Q.; Lee, J. Y.; Wang, D. I. C. The Synthesis of SERS-Active Gold Nanoflower Tags for in Vivo Applications. ACS Nano 2008, 2, 2473-2480.

(18) Khoury, C. G.; Vo-Dinh, T. Gold Nanostars for SurfaceEnhanced Raman Scattering: Synthesis, Characterization and Optimization. J. Phys. Chem. C 2008, 112, 18849-18859.

(19) Tian, L.; Tadepalli, S.; Fei, M.; Morrissey, J. J.; Kharasch, E. D.; Singamaneni, S. Off-Resonant Gold Superstructures as Ultrabright Minimally Invasive Surface-Enhanced Raman Scattering (SERS) Probes. Chem. Mater. 2015, 27, 5678-5684.

(20) Höller, R. P. M.; Dulle, M.; Thomä, S.; Mayer, M.; Steiner, A. M.; Förster, S.; Fery, A.; Kuttner, C.; Chanana, M. Protein-Assisted Assembly of Modular 3D Plasmonic Raspberry-like Core/Satellite Nanoclusters: Correlation of Structure and Optical Properties. ACS Nano 2016, 10, 5740-5750.

(21) Rong, Z.; Xiao, R.; Wang, C.; Wang, D.; Wang, S. Plasmonic Ag Core-Satellite Nanostructures with a Tunable Silica-Spaced Nanogap for Surface-Enhanced Raman Scattering. Langmuir 2015, 31, 81298137.

(22) Wang, K.; Jiang, L.; Zhang, F.; Wei, Y.; Wang, K.; Wang, H.; Qi, Z.; Liu, S. Strategy for In Situ Imaging of Cellular Alkaline Phosphatase Activity Using Gold Nanoflower Probe and Localized Surface Plasmon Resonance Technique. Anal. Chem. 2018, 90, 14056-14062.

(23) Feng, Y.; Wang, Y.; Song, X.; Xing, S.; Chen, H. Depletion Sphere: Explaining the Number of Ag Islands on Au Nanoparticles. Chem. Sci. 2017, 8, 430-436. 
(24) Feng, Y.; He, J.; Wang, H.; Tay, Y. Y.; Sun, H.; Zhu, L.; Chen, $\mathrm{H}$. An Unconventional Role of Ligand in Continuously Tuning of Metal-Metal Interfacial Strain. J. Am. Chem. Soc. 2012, 134, 20042007.

(25) Feng, Y.; Wang, Y.; He, J.; Song, X.; Tay, Y. Y.; Hng, H. H.; Ling, X. Y.; Chen, H. Achieving Site-Specificity in Multistep Colloidal Synthesis. J. Am. Chem. Soc. 2015, 137, 7624-7627.

(26) Mei, R.; Wang, Y.; Liu, W.; Chen, L. Lipid Bilayer-Enabled Synthesis of Waxberry-like Core-Fluidic Satellite Nanoparticles: Toward Ultrasensitive Surface-Enhanced Raman Scattering Tags for Bioimaging. ACS Appl. Mater. Interfaces 2018, 10, 23605-23616.

(27) Lim, B.; Kobayashi, H.; Yu, T.; Wang, J.; Kim, M. J.; Li, Z.-Y.; Rycenga, M.; Xia, Y. Synthesis of Pd-Au Bimetallic Nanocrystals via Controlled Overgrowth. J. Am. Chem. Soc. 2010, 132, 2506-2507.

(28) Fennell, J.; He, D.; Tanyi, A. M.; Logsdail, A. J.; Johnston, R. L.; Li, Z. Y.; Horswell, S. L. A Selective Blocking Method to Control the Overgrowth of Pt on Au Nanorods. J. Am. Chem. Soc. 2013, 135, 6554-6561.

(29) Smith, J. D.; Bunch, C. M.; Li, Y.; Koczkur, K. M.; Skrabalak, S. E. Surface Versus Solution Chemistry: Manipulating Nanoparticle Shape and Composition Through Metal-Thiolate Interactions. Nanoscale 2019, 11, 512-519.

(30) Lee, J.-H.; Kim, G.-H.; Nam, J.-M. Directional Synthesis and Assembly of Bimetallic Nanosnowmen with DNA. J. Am. Chem. Soc. 2012, 134, 5456-5459.

(31) Straney, P. J.; Diemler, N. A.; Smith, A. M.; Eddinger, Z. E.; Gilliam, M. S.; Millstone, J. E. Ligand-Mediated Deposition of Noble Metals at Nanoparticle Plasmonic Hotspots. Langmuir 2018, 34, 1084-1091.

(32) Zhang, W.; Liu, J.; Niu, W.; Yan, H.; Lu, X.; Liu, B. TipSelective Growth of Silver on Gold Nanostars for Surface-Enhanced Raman Scattering. ACS Appl. Mater. Interfaces 2018, 10, 1485014856.

(33) Lin, S.; Lin, X.; Liu, Y.; Zhao, H.; Hasi, W.; Wang, L. Selfassembly of Au@Ag Core-Shell Nanocubes Embedded with an Internal Standard for Reliable Quantitative SERS Measurements. Anal. Methods 2018, 10, 4201-4208.

(34) Khlebtsov, B. N.; Khanadeev, V. A.; Tsvetkov, M. Y.; Bagratashvili, V. N.; Khlebtsov, N. G. Surface-Enhanced Raman Scattering Substrates Based on Self-Assembled PEGylated Gold and Gold-Silver Core-Shell Nanorods. J. Phys. Chem. C 2013, 117, 23162-23171.

(35) Rice, D.; Mouras, R.; Gleeson, M.; Liu, N.; Tofail, S. A. M.; Soulimane, T.; Silien, C. APTES Duality and Nanopore Seed Regulation in Homogeneous and Nanoscale-Controlled Reduction of Ag Shell on SiO2 Microparticle for Quantifiable Single Particle SERS. ACS Omega 2018, 3, 13028-13035.

(36) Wu, Y.; Li, P.; Yang, L.; Liu, J. Individual SERS Substrate with Core-Satellite Structure Decorated in Shrinkable Hydrogel Template for Pesticide Detection. J. Raman Spectrosc. 2014, 45, 68-74.

(37) Gandra, N.; Singamaneni, S. Bilayered Raman-Intense Gold Nanostructures with Hidden Tags (BRIGHTs) for High-Resolution Bioimaging. Adv. Mater. 2013, 25, 1022-1027.

(38) Zhao, Y.; Zheng, F.; Ke, W.; Zhang, W.; Shi, L.; Liu, H. GapTethered Au@AgAu Raman Tags for the Ratiometric Detection of MC-LR. Anal. Chem. 2019, 91, 7162-7172.

(39) Cha, M. G.; Lee, S.; Park, S.; Kang, H.; Lee, S. G.; Jeong, C.; Lee, Y.-S.; Kim, C.; Jeong, D. H. A Dual Modal Silver Bumpy Nanoprobe for Photoacoustic Imaging and SERS Multiplexed Identification of in Vivo Lymph Nodes. Nanoscale 2017, 9, 1255612564.

(40) Shao, D.; Zhang, X.; Liu, W.; Zhang, F.; Zheng, X.; Qiao, P.; Li, J.; Dong, W.-f.; Chen, L. Janus Silver-Mesoporous Silica Nanocarriers for SERS Traceable and pH-Sensitive Drug Delivery in Cancer Therapy. ACS Appl. Mater. Interfaces 2016, 8, 4303-4308.

(41) Chen, M.; Zhang, L.; Gao, M.; Zhang, X. High-Sensitive Bioorthogonal SERS Tag for Live Cancer Cell Imaging by SelfAssembling Core-Satellites Structure Gold-Silver Nanocomposite. Talanta 2017, 172, 176-181.
(42) Zeng, Y.; Ren, J.-Q.; Shen, A.-G.; Hu, J.-M. Splicing Nanoparticles-Based "Click" SERS Could Aid Multiplex Liquid Biopsy and Accurate Cellular Imaging. J. Am. Chem. Soc. 2018, 140, 10649-10652.

(43) Chen, Y.; Ren, J.-Q.; Zhang, X.-G.; Wu, D.-Y.; Shen, A.-G.; Hu, J.-M. Alkyne-Modulated Surface-Enhanced Raman Scattering-Palette for Optical Interference-Free and Multiplex Cellular Imaging. Anal. Chem. 2016, 88, 6115-6119.

(44) Li, S.; Chen, T.; Wang, Y.; Liu, L.; Lv, F.; Li, Z.; Huang, Y.; Schanze, K. S.; Wang, S. Conjugated Polymer with Intrinsic Alkyne Units for Synergistically Enhanced Raman Imaging in Living Cells. Angew. Chem., Int. Ed. 2017, 56, 13455-13458.

(45) Jin, Q.; Fan, X.; Chen, C.; Huang, L.; Wang, J.; Tang, X. Multicolor Raman Beads for Multiplexed Tumor Cell and Tissue Imaging and in Vivo Tumor Spectral Detection. Anal. Chem. 2019, 91, 3784-3789.

(46) Hahm, E.; Cha, M. G.; Kang, E. J.; Pham, X.-H.; Lee, S. H.; Kim, H.-M.; Kim, D.-E.; Lee, Y.-S.; Jeong, D.-H.; Jun, B.-H. Multilayer Ag-Embedded Silica Nanostructure as a Surface-Enhanced Raman Scattering-Based Chemical Sensor with Dual-Function Internal Standards. ACS Appl. Mater. Interfaces 2018, 10, 40748-40755. 